\title{
Electromagnetically Induced Transparency Using a Artificial Molecule in Circuit Quantum Electrodynamics
}

\author{
Hai-Chao Li, Guo-Qin Ge \\ School of Physics, Huazhong University of Science and Technology, Wuhan, China \\ Email: 1hc2007@hust.edu.cn,gqge@hust.edu.cn
}

Received 2013

\begin{abstract}
Electromagnetically induced transparency (EIT) having wide applications in quantum optics and nonlinear optics is explored ordinarily in various atomic systems. In this paper we present a theoretical study of EIT using supercon- ducting circuit with a V-type artificial molecule constructed by two Josephson charge qubits coupled each other through a large capacitor. In our theoretical model we make a steady state approximation and obtain the analytical expressions of the complex susceptibility for the artificial system via the density matrix formalism. The complex susceptibility has additional dependence on the qubit parameters and hence can be tuned to a certain extent.
\end{abstract}

Keywords: EIT; Artificial Molecule; Complex Susceptibility

\section{Introduction}

Electromagnetically induced transparency (EIT) [1,2] through quantum coherent effects has attracted considerable interest due to its extensive applications in quantum optics and atomic physics. The first experimental demonstration of EIT was based on a $\Lambda$-type atomic system [3]. EIT has also been observed experimentally in the Vtype [4] and cascade-type [5] energy level configurations. It's of particular interest to indicate EIT how to appear via quantum interference in a $\mathrm{V}$-type system because population trapping isn't involved. In contrast to the usual weak probe regime, EIT can be realized in the strong probe regime [6], where population inversion is not correlated with optical gain and the traditional corresponddence between inversion and gain is not satisfied.

Circuit quantum electrodynamics(QED) $[7,8]$, where transmission line resonator plays the role of cavity and superconducting qubit $[9,10]$ behaves as artificial atom to replace the natural atom, has recently become a new testbed for quantum optics. Compared with the conventional cavity QED with atomic gases, superconducting circuits as artificial quantum systems in solid-state devices have significant advantages, such as offering long coherence time to implement the quantum gate operations [11], huge tunability and controllability by external electromagnetic fields [12]. As an on-chip realization of cavity QED, circuit QED has reproduced many quantum optical phenomena, including Kerr and cross-Kerr nonlinearities [12,13], the Mollow Triplet [14], Autler-Townes effect [15], EIT [16, 17]. Further- more, circuit QED can be used to realize ultrastrong coupling regime [18] previously inaccessible to atomic systems and explore novel optical phenomena emerging only in this regime.

Although have being extensively studied in traditional atomic systems, investigations of EIT phenomena in superconducting circuits based on mesoscopic Josephson junctions are still scarce. Recently experimental observation of EIT has been reported by using a single artificial atom coupled to a 1D transmission line [16] and EIT can be utilized as a sensitive probe of decoherence in superconducting circuits [19]. Besides, a nanomechanical resonator can provide additional auxiliary energy levels to a superconducting Cooper-pair box so that EIT can be realized in the system [20].

Motivated by these investigations, we propose a scheme to perform EIT employing V-type artificial molecule, which is constructed by two superconducting charge qubits coupled each other through a large capacitor. In our EIT scheme, a weak probe field with Rabi frequency $\Omega_{1}$ and frequency $\omega_{1}$ couples the $|1\rangle \leftrightarrow|3\rangle$ transition while a strong control field with Rabi frequency $\Omega_{2}$ and frequency $\omega_{2}$ couples the $|1\rangle \leftrightarrow|2\rangle$ transition, as shown in Figure 1.

This paper is organized as follows. We first describe the theoretical model and gain the energy spectrum of the V-type artificial system in Section 2. Then, we give steady-state analysis of EIT by utilizing the density matrix method and acquire the complex susceptibility for the superconducting system in Section 3 and our conclusions are given in Section 4. 


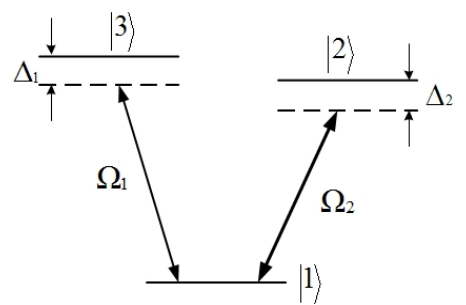

Figure 1. Schematic illustration of EIT for the artificial molecule.

\section{The Model of Artificial Molecule}

Let us consider two interacting superconducting charge qubits which are electrostatically coupled to each other by a large capacitor $C_{m}$. Each charge qubit has a superconducting quantum interference device (SQUID) ring geometry biased by an external flux and so the effective Josephson coupling energy can be varied from zero up to its maximum value. The Hamiltonian of coupled qubits reads

$$
\begin{aligned}
H= & E_{c 1}\left(n_{1}-n_{g 1}\right)^{2}-E_{J 1} \cos \beta_{1}+E_{c 2}\left(n_{2}-n_{g 2}\right)^{2} \\
& -E_{J 2} \cos \beta_{2}+E_{m}\left(n_{1}-n_{g 1}\right)\left(n_{2}-n_{g 2}\right)
\end{aligned}
$$

The first four terms represent two independent qubits and the last term describes the interaction between the qubits due to the electrostatical coupling of the capacitor. $E_{J 1}$ and $E_{J 2}$ are the effective Josephson coupling energy for the corresponding SQUID; $\beta_{1}$ and $\beta_{2}$ are the phases of the SQUID; $E_{c 1}$ and $E_{c 2}$ are the effective Cooper-pair charging energies for the qubits; $n_{i}$ and $n_{g i}$ for $I=1,2$ are the number operator of excess Cooper-pairs on the island and the normalized gate induced charge; $E_{m}$ is the capacitive coupling energy between the charge qubits.

Working in the vicinity of one degeneracy point $\left(n_{g i} \in\right.$ $[0,1])$, only two adjacent charge states $|0\rangle$ and $|1\rangle$ on the island are relevant while all other charge states, having a much higher energy, can be ignored [10]. In this case the Hamiltonian can be written as

$$
\begin{aligned}
H= & \sum_{i=1,2}\left(-\frac{1}{2} B_{z i} \sigma_{z i}-\frac{1}{2} E_{J i} \sigma_{x i}\right)+\frac{1}{4} E_{m} \sigma_{z 1} \sigma_{z 2} \\
& -\frac{1}{2} E_{m} \delta_{2} \sigma_{z 1}-\frac{1}{2} E_{m} \delta_{1} \sigma_{z 2}
\end{aligned}
$$

where $B_{z i}=E_{c i}\left(1-2 n_{g i}\right)$ for $I=1,2$ are the difference of the electrostatic energy between the states $|0\rangle$ and $|1\rangle$, $\sigma_{z}$ and $\sigma_{x}$ are the Pauli matrices and $\delta_{i}=1 / 2-n_{g i}$. Switching to the eigenbasis $|e\rangle$ and $|g\rangle$ of the qubits and exactly at the co-resonance point $\delta_{i}=0$, the Hamiltonian takes the form

$$
H=\frac{1}{2} E_{J 1} \rho_{z 1}+\frac{1}{2} E_{J 2} \rho_{z 2}+\frac{1}{4} E_{m} \rho_{x 1} \rho_{x 2}
$$

To avoid confusion we introduce a second set of Pauli operator $\boldsymbol{\rho}$ acting on the eigenstates of qubits. Without loss of generality, we assume that the two superconducting charge qubits are identical (i.e., $E_{c 1}=E_{c 2}=E_{c}$, $\left.E_{J 1}=E_{J 2}=E_{J}\right)$. So the eigenvalues of coupled qubits are readily written as

$$
\begin{aligned}
& E_{4}=\frac{1}{4}\left(E_{m}^{2}+16 E_{J}^{2}\right)^{\frac{1}{2}} \\
& E_{3}=\frac{1}{4} E_{m} \\
& E_{2}=-\frac{1}{4} E_{m} \\
& E_{1}=-\frac{1}{4}\left(E_{m}^{2}+16 E_{J}^{2}\right)^{\frac{1}{2}}
\end{aligned}
$$

with the corresponding eigenstates being

$$
\begin{aligned}
& |4\rangle=\sin \alpha|g g\rangle+\cos \alpha|e e\rangle \\
& |3\rangle=\frac{1}{\sqrt{2}}(|g e\rangle+|e g\rangle) \\
& |2\rangle=\frac{1}{\sqrt{2}}(|g e\rangle-|e g\rangle) \\
& |1\rangle=\cos \alpha|g g\rangle-\sin \alpha|e e\rangle
\end{aligned}
$$

Here the parameter $\alpha$ satisfies the following relations

$$
\sin 2 \alpha=\frac{E_{m}}{\sqrt{E_{m}^{2}+16 E_{J}^{2}}} \quad \cos 2 \alpha=\frac{4 E_{J}}{\sqrt{E_{m}^{2}+16 E_{J}^{2}}}
$$

It is worthwhile to note that arbitrary transitions can not be allowed in the above four states due to selection rules for superconducting qubits. By calculating the matrix elements of $\rho_{x 1}$ and $\rho_{x 2}$ between the eigenstates, we find that the transitions $|1\rangle \leftrightarrow|4\rangle$ and $|2\rangle \leftrightarrow|3\rangle$ are forbidden while the other transitions with nonzero matrix elements are allowed. choosing the three levels with lowest eigenenergies shown in Figure 1, we obtain the Vtype artificial system.

\section{Complex Susceptibility}

EIT phenomenon of a closed three level system interacting with a weak probe field and a strong control field can be demonstrated by adopting the density matrix formalism. In the eigenbasis of the qubits, the interaction Hamiltonian between the three-level artificial molecule and two semiclassical fields is expressed as $(\hbar=1)$

$$
H_{\text {int }}=-\frac{1}{2}\left(\Omega_{1} e^{-i \omega_{1} t}|e\rangle_{11}\left\langle g\left|+\Omega_{2} e^{-i \omega_{2} t}\right| e\right\rangle_{22}\langle g|+H . c .\right)
$$

In the basis $\{|1\rangle,|2\rangle$ and $|3\rangle\}$ of the V-type artificial system and with the rotating-wave approximation, the interaction Hamiltonian is given by

$$
\begin{aligned}
H_{\text {int }}= & -\frac{1}{2}\left(\Omega_{1} e^{-i \omega_{1} t} \frac{1}{\sqrt{2}} \cos \alpha|3\rangle\langle 1|\right. \\
& \left.+\Omega_{2} e^{-i \omega_{2} t} \frac{1}{\sqrt{2}} \cos \alpha|2\rangle\langle 1|+\text { H.c. }\right)
\end{aligned}
$$


In the interaction picture, the Hamiltonian of the system reads

$$
H_{I}=\Delta_{1}|3\rangle\left\langle 3\left|+\Delta_{2}\right| 2\right\rangle\langle 2|-\frac{1}{2}\left(\xi_{1}|3\rangle\left\langle 1\left|+\xi_{2}\right| 2\right\rangle\langle 1|+\text { H.c. }\right) \text { (9) }
$$

where $\Delta_{1}=\omega_{31} \omega_{1}$ is the detuning of the probe field, $\Delta_{2}=$ $\omega_{21} \omega_{2}$ is the detuning of the control field,

$$
\xi_{1}=\Omega_{1} \cos \alpha / \sqrt{2} \text { and } \xi_{2}=\Omega_{2} \cos \alpha / \sqrt{2} .
$$

We can select the frequencies of the fields so that the probe field $\omega_{1}$ and the control field $\omega_{2}$ are near resonant with the transitions $|1\rangle \leftrightarrow|3\rangle$ and $|1\rangle \leftrightarrow|2\rangle$, respectively. In this case, other transitions can be ignored in our discussion. The evolution of the system is governed by the set of density matrix equations of motion

$$
\begin{aligned}
& \dot{\rho}_{33}=-\Gamma_{3} \rho_{33}+\frac{i}{2} \xi_{1} \rho_{13}-\frac{i}{2} \xi_{1}^{*} \rho_{31} \\
& \dot{\rho}_{22}=-\Gamma_{2} \rho_{22}+\frac{i}{2} \xi_{2} \rho_{12}-\frac{i}{2} \xi_{2}^{*} \rho_{21} \\
& \dot{\rho}_{31}=-\left(\gamma_{31}+i \Delta_{1}\right) \rho_{31}+\frac{i}{2} \xi_{1}\left(\rho_{11}-\rho_{33}\right)-\frac{i}{2} \xi_{2} \rho_{32} \\
& \dot{\rho}_{21}=-\gamma_{21} \rho_{21}+\frac{i}{2} \xi_{2}\left(\rho_{11}-\rho_{22}\right)-\frac{i}{2} \xi_{1} \rho_{23} \\
& \dot{\rho}_{32}=-\left(\gamma_{32}+i \Delta_{1}\right) \rho_{32}-\frac{i}{2} \xi_{2}^{*} \rho_{31}+\frac{i}{2} \xi_{1} \rho_{12} \\
& 1=\rho_{11}+\rho_{22}+\rho_{33}
\end{aligned}
$$

Here we further assume that the control field frequency $\omega_{c}$ matchs the level spacing between the states $|2\rangle$ and $|1\rangle$, i.e. $\Delta_{2}=0$. In these equations we have introduced phenomenologically the relaxation rates $\Gamma_{i}(I=1,2,3)$ for the levels as well as the total dephasing rates $\gamma_{i j}=$ $\left(\Gamma_{i}+\Gamma_{i}\right) / 2+\tau_{\varphi}$ including the relaxation and pure dephasing processes. Since we are interested in the dispersion and absorption properties of the V-type artificial system, only first-order perturbation expansion of the equations of matrix elements are necessary. For the system we set[21]

$$
\rho_{33}^{(0)}=0 \quad \rho_{22}^{(0)}=\frac{B}{\Gamma_{2}+2 B} \rho_{11}^{(0)}=\frac{\Gamma_{2}+B}{\Gamma_{2}+2 B}
$$

where $B$ is the rate of pumping by the control field

$$
B=\frac{1}{2} \frac{\left|\Omega_{2}\right|^{2}}{\gamma_{21}}
$$

Taking into account the steady-state solution (i.e., all derivatives are set equal to zero), we have the first order matrix element

$$
\begin{aligned}
\rho_{31}^{(1)}= & \frac{i \xi_{1}}{2}\left[\rho_{11}^{(0)}\left(\gamma_{32}+i \Delta_{1}\right)-\frac{\left|\xi_{2}\right|^{2}\left(\rho_{11}^{(0)}-\rho_{22}^{(0)}\right)}{4 \gamma_{21}}\right] \\
& \times\left[\left(\gamma_{31}+i \Delta_{1}\right)\left(\gamma_{32}+i \Delta_{1}\right)+\frac{\left|\xi_{2}\right|^{2}}{4}\right]^{-1}
\end{aligned}
$$

Combining the relation

$$
\varepsilon_{0} \chi \Omega_{1}=2\left|\mu_{31}\right|^{2} \rho_{31}
$$

with equation (12), we have the following expressions of the complex susceptibility $\chi=\chi^{\prime}+i \chi^{\prime \prime}$ :

$$
\begin{gathered}
\chi^{\prime}=-\frac{\cos \alpha\left|\mu_{31}\right|^{2} \Delta_{1}}{\sqrt{2} \varepsilon_{0} Z}\left[\rho_{11}^{(0)}\left(\frac{\left|\xi_{2}\right|^{2}}{4}-\Delta_{1}^{2}-\gamma_{32}^{2}\right)\right. \\
\left.+\frac{\left|\xi_{2}\right|^{2}}{4 \gamma_{21}}\left(\rho_{11}^{(0)}-\rho_{22}^{(0)}\right)\left(\gamma_{31}+\gamma_{32}\right)\right] \\
\chi^{\prime \prime}=\frac{\cos \alpha\left|\mu_{31}\right|^{2}}{\sqrt{2} \varepsilon_{0} Z}\left\{\rho_{11}^{(0)}\left[\gamma_{32}\left(\gamma_{31} \gamma_{32}+\frac{\left|\xi_{2}\right|^{2}}{4}\right)+\gamma_{31} \Delta_{1}^{2}\right]\right. \\
\left.-\frac{\left|\xi_{2}\right|^{2}}{4 \gamma_{21}}\left(\rho_{11}^{(0)}-\rho_{22}^{(0)}\right)\left(\frac{\left|\xi_{2}\right|^{2}}{4}+\gamma_{31} \gamma_{32}-\Delta_{1}^{2}\right)\right\}
\end{gathered}
$$

where $\varepsilon_{0}$ is the vacuum permittivity, $\mu_{31}$ is the transition dipole moment and

$$
Z=\left(\frac{\left|\xi_{2}\right|^{2}}{4}+\gamma_{31} \gamma_{32}-\Delta_{1}^{3}\right)^{2}\left(\gamma_{31} \Delta_{1}+\gamma_{32} \Delta_{1}\right)^{2}
$$

It seems that the above expressions are similar to the susceptibilities of the conventional three level atomic systems, but here the complex susceptibility of the artificial molecule has additional dependence on the tunable Josephson coupling energy $E_{J}$ and the capacitive coupling strength $E_{m}$ through the parameter $\alpha$ and hence can be tuned to a certain extent.

Figures 2(a) and (b) plot both the real and imaginary parts of the susceptibility $\chi$ as a function of the probe detuning $\Delta_{1}$ and the dimensionless ratio of the Josephson coupling energy $E_{J}$ to the interaction energy $E_{m}$ according to the equations (15) and (16). Figure 2 shows that the absorption profile is even symmetric and the dispersion profile is odd about the zero probe detuning point $\Delta_{1}$ $=0$. We can observe that the absorption is minimum at the zero point $\Delta_{1}=0$ and increases with the growth of the Josephson coupling energy $E_{J}$, but the absorption value does not become large after the $E_{J}$ is increased to a cer- tain value, as can be seen from Figure 2(b).

Moreover, absorption coefficient can be dominated greatly by the control field strength $\Omega_{c}$, as depicted in Figure 3. From the drawing, we see that single absorption peak appears in the regime of weak control field and indicates strong absorption to probe field. As the control power is increased, the doublet spacing of absorption curve increases and absorption value between the two peaks gradually tends to zero EIT, i.e. EIT effect arises in the higher control intensities.

\section{Conclusions}

In conclusion, we have theoretically investigated the EIT 

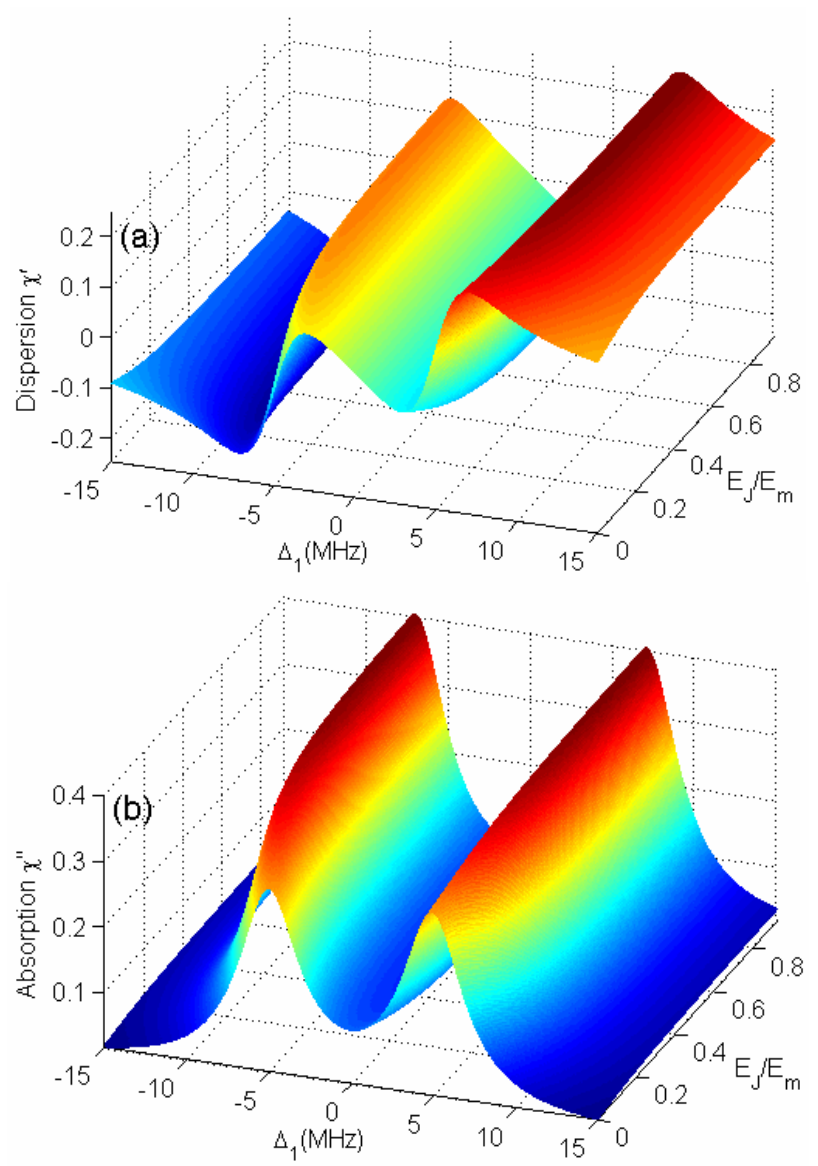

Figure 2. Real part $\chi^{\prime}$ and imaginary part $\chi^{\prime \prime}$ of the complex susceptibility versus the probe detuning $\Delta_{1}$ and the dimensionless ratio $\mathrm{E}_{\mathrm{J}} / \mathbf{E}_{\mathrm{m}}$. Here parameters $E_{J \max }=14.5 \mathrm{GHz}, E_{m}=$ 15.7GHz, $\gamma_{31}=\gamma_{21}=2 \mathrm{MHz}, \gamma_{32}=2.5 \mathrm{MHz}, \Gamma_{2}=1 / 0.7 \mathrm{MHz}, \Omega_{c}$ $=20 \mathrm{MHz}$.

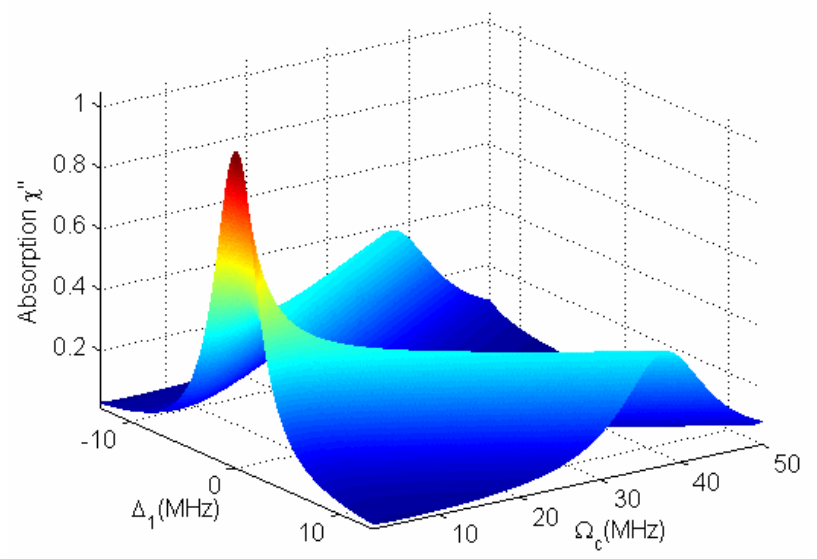

Figure 3. Absorption coefficient $\chi^{\prime \prime}$ as a function of the probe detuning $\Delta_{1}$ and the control field intensity $\Omega_{c}$ (from 2 to $50 \mathrm{MHz}$ ). Parameters $E_{J}=14 \mathrm{GHz}, E_{m}=15.7 \mathrm{GHz}, \gamma_{31}=$ $\gamma_{21}=2 \mathrm{MHz}, \gamma_{32}=2.5 \mathrm{MHz}, \Gamma_{2}=1 / 0.7 \mathrm{MHz}$.

effect in a V-type artificial system derived from two coupled superconducting charge qubits. Using the density ma- trix formalism, we obtain the analytical expressions of the complex susceptibility which have extra dependence on qubit parameters $E_{J}$ and $E_{m}$. As a result, EIT can be tuned to a certain extent by changing the Josephson coupling energy $E_{J}$ compared with the conventional EIT phenomenon in the atomic systems where atomic parameters are uaually fixed.

\section{Acknowledgements}

This work was supported in part by the National Natural Science Foundation of China under the Grant No. 11 274132 and the Nature Science Foundation of Hubei Province.

\section{REFERENCES}

[1] D. J. Fulton, S. Shepherd, R. R. Moseley, B. D. Sinclair, and M. H. Dunn, "Continuous-wave Electromagnetically Induced Transparency: a Comparison of $\mathrm{V}, \Lambda$, and Cascade Systems," Physical Review A, Vol. 52, No. 3, 1995, pp. 2302-2311. doi: 10.1103/PhysRevA.52.2302

[2] M. Fleischhauer, A. Imamoglu and J. P. Marangos, "Electromagnetically Induced Transparency: Optics in Coherent Media," Reviews of Modern Physics, Vol. 77, No. 2, 2005, pp. 633-673.

doi:10.1103/RevModPhys.77.633

[3] K. J. Boller, A. Imamoglu and S. E. Harris, "Observation of Electromagnetically Induced Transparency," Physical Review Letters, Vol. 66, No. 20, 1991, pp. 2593-2596. doi: 10.1103/PhysRevLett.66.2593

[4] A. Lazoudis, T. Kirova, E. H. Ahmed, P. Qi, J. Huennekens and A. M. Lyyra, "Electromagnetically Induced Transparency in an Open V-type Molecular System," Physical Review A, Vol. 83, No. 6, 2011, pp. 063419.

doi: 10.1103/PhysRevA.83.063419

[5] J. Gea-Banacloche, Y. Q. Li, S. Z. Jin and Min Xia, "Electromagnetically Induced Transparency in Laddertype Inhomogeneously Broadened Media: Theory and Experiment," Physical Review A, Vol. 51, No. 1, 1995, pp. 576-584. doi: 10.1103/PhysRevA.51.576

[6] S. Wielandy and A. L. Gaeta, "Investigation of electromagnetically induced transparency in the strong probe regime," Physical Review A, Vol. 58, No. 3, 1998, pp. 2500-2505. doi: 10.1103/PhysRevA.58.2500

[7] J. Q. You and F. Nori, "Atomic Physics and Quantum Optics Using Superconducting Circuits," Nature, Vol. 474, No. 7353, 2011, pp. 589-597. doi: $10.1038 /$ nature 10122

[8] S. M. Girvin, M. H. Devoret and R. J. Schoelkopf, "Circuit QED and Engineering Charge-based Superconducting Qubits," Physica Scripta, Vol. 2009, No. T137, 2009, pp. 014012.

doi:10.1088/0031-8949/2009/T137/014012

[9] J. Q. You and F. Nori, "Superconducting Circuits and Quantum Information," Physics Today, Vol. 58, No. 11, 2005, pp. 42-47. doi: 10.1063/1.2155757 
[10] Y. A. Pashkin, O. Astafiev, T. Yamamoto, Y. Nakamura and J. S. Tsai, "Josephson Charge Qubits: a Brief Review," Quantum Information Processing, Vol. 8, 2009, pp. 55-80. doi: 10.1007/s11128-009-0101-5

[11] B. C. Sanders, "Quantum Optics in Superconducting Circuits," AIP Conference Proceedings, Vol. 1398, 2011, pp. 46-49. doi: 10.1063/1.3644209

[12] Y. Hu, G. Q. Ge, S. Chen, X. F. Yang and Y. L. Chen, "Cross-Kerr-effect Induced by Coupled Josephson Qubits in Circuit Quantum Electrodynamics," Physical Review A, Vol. 84, No. 1, 2011, p. 012329. doi: 10.1103/PhysRevA.84.012329

[13] S. Rebić, J. Twamley and G. J. Milburn, "Giant Kerr Nonlinearities in Circuit Quantum Electrodynamics," Physical Review Letters, Vol. 103, No. 15, 2009, p. 150503. doi: 10.1103/PhysRevLett.103.150503

[14] O. Astafiev et al., "Resonance Fluorescence of a Single Artificial Atom," Science, Vol. 327, No. 5967, 2010, pp. 840-843. doi: 10.1126/science. 1181918

[15] M. A. Sillanpää et al., "Autler-Townes Effect in a Superconducting Three-Level System," Physical Review Letters, Vol. 103, No. 19, 2009, p. 193601. doi: 10.1103/PhysRevLett.103.193601

[16] A. A. Abdumalikov, Jr., O. Astafiev, A. M. Zagoskin, Yu. A. Pashkin, Y. Nakamura and J. S. Tsai, "Electromagnetically Induced Transparency on a Single Artificial
Atom," Physical Review Letters, Vol. 104, No. 19, 2010, p. 193601. doi: 10.1103/PhysRevLett.104.193601

[17] J. Joo, J. Bourassa, A. Blais and B. C. Sanders, "Electromagnetically Induced Transparency with Amplification in Superconducting Circuits," Physical Review Letters, Vol. 105, No. 7, 2010, p. 073601.

doi: 10.1103/PhysRevLett.105.073601

[18] T. Niemczyk et al., "Circuit Quantum Electrodynamics in the Ultrastrong-coupling Regime," Nature Physics, Vol. 6, No. 10, 2010, pp. 772-776. doi: 10.1038/nphys 1730

[19] K. V. R. M. Murali, Z. Dutton, W. D. Oliver, D. S. Crankshaw and T. P. Orlando, "Probing Decoherence with Electromagnetically Induced Transparency in Superconductive Quantum Circuits," Physical Review Letters, Vol. 93, No. 8, 2004, pp. 087003. doi: 10.1103/PhysRevLett.93.087003

[20] X. Z. Yuan, H. S. Goan, C. H. Lin, K. D. Zhu and Y. W. Jiang, "Nanomechanical-resonator-assisted Induced Transparency in a Cooper-pair Box System," New Journal of Physics, Vol. 10, 2008, p. 095016. doi: 10.1088/1367-2630/10/9/095016

[21] G. R. Welch, "Observation of V-Type Electromag- netically Induced Transparency in a Sodium Atomic Beam," Foundations of Physics, Vol.28, No. 4, 1998, pp. 621-638. doi: 10.1023/A:1018765706887 